\title{
É necessária a realização de biópsia de medula óssea bilateral para 0 estadiamento do linfoma difuso de grandes células B?
}

\author{
Is it necessary to perform a bilateral bone marrow biopsy to stage diffuse large B-cell lymphoma?
}

Marcelo Bellesso'; Rodrigo Dolphini Velasques²; Luis Fernando Pracchia'; Beatriz Beitler³ ${ }^{3}$ Vera Lúcia Aldred ${ }^{4}$; Dalton Alencar Fisher Chamone ${ }^{5}$; Juliana Pereira ${ }^{6}$

\author{
unitermos \\ Linfoma não-Hodgkin \\ Estadiamento \\ Biópsia de medula óssea
}

\section{resumo}

Estudo retrospectivo que visa analisar a utilidade da biópsia de medula óssea (BMO) bilateral na infiltração de medula óssea (MO) por linfoma difuso de grandes células B (LDGCB). Nossos objetivos foram avaliar a incidência de infiltração unilateral de MO por LDGCB e comparar o comprimento dos fragmentos obtidos entre as amostras positivas e negativas para infiltração. Além disso, verificamos se houve diferença entre os casos com infiltração unilateral versus bilateral, correlacionando com desidrogenase láctica (DHL) e estadiamento tomográfico. Avaliamos 268 casos de LDGCB e observamos infiltração medular em 34 casos (13\%). Não foi possível a avaliação de seis casos, restando 28 casos para análise. Foram revisados no total 70 fragmentos de $\mathrm{MO}$ sobre presença ou ausência de infiltração e comprimento. A média do número de fragmentos por casos foi 2,5; a média do comprimento dos fragmentos foi $11,01 \mathrm{~mm}( \pm 5,12 \mathrm{~mm})$, e a média do comprimento dos fragmentos por caso foi $27,53 \mathrm{~mm}$. Foi observado que em seis casos $(21,4 \%)$ havia infiltração unilateral. Não foram evidenciadas diferenças nas médias do comprimento dos fragmentos em relação à presença versus ausência de infiltração $10,95 \mathrm{~mm}( \pm 5,1 \mathrm{~mm})$ versus $11,57 \mathrm{~mm}( \pm 5,2 \mathrm{~mm})$, $p>0,05$, respectivamente. Não foram evidenciadas diferenças em 23 casos entre a comparação da infiltração medular unilateral versus bilateral com DHL e estadiamento tomográfico. Concluímos que a BMO bilateral foi superior à unilateral, pois pode aumentar a detecção de infiltração de $\mathrm{MO}$ em 21,4\% dos casos.

\section{abstract}

This retrospective study aims to analyze the usefulness of bilateral bone marrow biopsy in bone marrow infiltration by diffuse large B-cell lymphoma ( $D L B C L)$. Our objectives were to assess the incidence of unilateral $B M$ involvement by $D L B C L$ and compare fragment length obtained from positive and negative samples for infiltration. Furthermore, we compared the differences between unilateral and bilateral infiltration correlating with lactic dehydrogenase (LDH) and computerized tomography (CT) staging. We evaluated 268 cases of $D L B C L$ and observed medullary infiltration in 34 cases (13\%). It was not possible to evaluate 6 out of 34 cases. 70 BM fragments were reviewed as to the presence or absence of infiltration and length. The mean number of fragments per case was 2.5; the mean BM fragment length was $11.01 \mathrm{~mm}( \pm 5.12 \mathrm{~mm})$ and the mean BM fragment length per case was $27.53 \mathrm{~mm}$. There was unilateral BM infiltration in six cases (21.4\%). There were no differences in the mean fragment length as to the presence/absence of infiltration $10.95 \mathrm{~mm}$ ( $\pm 5.2 \mathrm{~mm}$ ) versus $11.57 \mathrm{~mm}, \mathrm{p}>0.05$, respectively. There were no differences in 23 cases between the comparison of unilateral medullary infiltration versus bilateral with lactic dehydrogenase and CT staging. We concluded that bilateral bone marrow biopsy was superior to unilateral because it may increase by $21.4 \%$ the detection of $B M$ involvement by $D L B C L$. key words

Non-Hodgkin lymphoma

Staging

Bone marrow biopsy

1. Médico hematologista; mestre pela Faculdade de Medicina da Universidade de São Paulo (FMUSP).

2. Médico hematologista.

3. Doutora pela FMUSP.

4. Médica patologista; mestra pela Fundação Antônio Prudente - Hospital do Câncer A. C. Camargo.

5. Professor titular de Hematologia e Hemoterapia da FMUSP.

6. Doutora pela FMUSP. 


\section{Introdução}

A infiltração de medula óssea ocorre de forma variada nos diversos tipos de linfoma não-Hodgkin (LNH). São mais frequentes nos linfomas indolentes comparados aos $\mathrm{LNH}$ agressivos. Cerca de $15 \%$ a $36 \%$ dos LNH agressivos apresentam infiltração medular, enquanto nos $\mathrm{LNH}$ indolentes a taxa da infiltração é de $40 \%$ a $90 \%{ }^{(2,5)}$. O diagnóstico do envolvimento medular no $\mathrm{LNH}$ promove informações importantes em relação ao prognóstico e ao plano terapêutico a ser estabelecido. A biópsia de medula óssea (BMO), associada aos métodos de imagem tomográficos, consiste em exames fundamentais para o estadiamento dos LNH. Embora seja utilizada a BMO como exame padrão para avaliação de infiltração tumoral, há outros exames capazes de diagnosticar a infiltração medular, com sensibilidades diferentes, como imunofenotipagem de aspirado de medula óssea (MO), tomografia com emissão de positrons (PET scan) e ressonância nuclear magnética (RNM). Poucos artigos foram publicados em relação às evidências de qual a melhor forma de se avaliar o envolvimento medular; entretanto, em 1999, foi sugerido que o fragmento mínimo aceitável seria de $20 \mathrm{~mm}^{(4)}$. Não há consenso perante a realização de $\mathrm{BMO}$ unilateral versus bilateral, mas publicações recentes sugerem que o comprimento do fragmento pode ser mais significativo que o número de sítios estudados ${ }^{(1)}$. Assim, elaboramos estudo retrospectivo que visa avaliar a necessidade da realização da BMO bilateral, como também comparar diferenças dos comprimentos dos fragmentos com a positividade de infiltração medular por linfoma difuso de grandes células B (LDGCB).

\section{Objetivo}

Avaliar a necessidade da realização de BMO bilateral para estadiamento do LDGCB por meio de análise das frequências de casos com infiltração unilateral, comparação das diferenças dos comprimentos dos fragmentos de $\mathrm{MO}$, correlacionando com a positividade de infiltração, e comparação dos casos de infiltrações unilateral e bilateral com marcador bioquímico e estadiamento radiológico.

\section{Casuística e metodologia}

Foram avaliados retrospectivamente casos de LDGCB tratados no período 2002 a 2006 no Serviço de Hemato- logia e Hemoterapia do Hospital das Clínicas da Faculdade de Medicina da Universidade de São Paulo (FMUSP). Os critérios de inclusão foram pacientes com LDGCB que sofreram BMO bilateral para o estadiamento e com infiltração de MO, constatada em pelo menos um dos fragmentos. Os casos selecionados foram revisados pelo Serviço de Anatomia-Patológica do Hospital das Clínicas da FMUSP. A revisão consistia em medir cada fragmento, avaliando isoladamente infiltração por LDGCB. Comparamos as médias dos fragmentos positivos e negativos por meio do teste estatístico Mann-Whitney, sendo considerada diferença estatística, $p<0,05$. A média do comprimento dos fragmentos de $\mathrm{MO}$ foi calculada através da somatório do comprimento de $\mathrm{MO}$ de todos os casos divididos pelo número de fragmentos. A média do comprimento de fragmentos de $\mathrm{MO}$ por caso foi obtida pela somatória do comprimento de todos os casos dividida pelo número de casos.

As comparações entre o envolvimento medular unilateral e o bilateral com as categorias estadiamento radiológico (I-II ou III-IV) e DHL (normal ou elevado) foram feitas pelo teste exato de Fisher. Foi utilizado software SPSS versão 10.0 .

\section{Resultados}

Entre 268 casos de LDGCB revisados, foi evidenciada infiltração de $\mathrm{MO}$ em 34 (13\%). Não foi possível revisão de seis casos. Assim, foram revisados 28, com média de idade de 62,18 anos, sendo $45 \%$ do sexo masculino. No total, foram avaliadas 70 amostras de fragmento de MO. A média de fragmentos por caso foi 2,5 . A variação dos comprimentos dos fragmentos de $\mathrm{MO}$ foi de 4 a $22 \mathrm{~mm}$. A média do comprimento dos fragmentos de $\mathrm{MO}$ foi 11,01 $\mathrm{mm}( \pm 5,12)$. A média do comprimento dos fragmentos por caso foi $27,53 \mathrm{~mm}$.

Em seis casos $(21,4 \%)$ foi constatada infiltração unilateral. Não foi evidenciada diferença estatística entre as médias do comprimento dos fragmentos positivo versus negativo: $10,95 \mathrm{~mm}( \pm 5,1) \times 11,57 \mathrm{~mm}( \pm 5,2), p=0,854$, respectivamente.

Foi possível analisar em 23 casos o estudo das comparações das categorias do estadiamento radiológico e DHL, comparados ao envolvimento bilateral ou unilateral da MO (Tabela). Não foi observada associação entre infiltração unilateral versus bilateral comparada às categorias 


\begin{tabular}{|c|c|c|c|}
\hline \multirow{2}{*}{$\begin{array}{l}\text { Tabela } \\
\text { Categorias }\end{array}$} & \multicolumn{3}{|c|}{$\begin{array}{l}\text { Distibuição dos } 23 \text { casos com } \\
\text { infiltração medular por LDGCB em } \\
\text { relação ao estadiamento tomográfico } \\
\text { e dosagens de DHL }\end{array}$} \\
\hline & $\begin{array}{c}\text { Infiltração } \\
\text { unilateral } \\
(n / \%)\end{array}$ & $\begin{array}{c}\text { Infiltração } \\
\text { bilateral } \\
(n / \%)\end{array}$ & $p$ \\
\hline \multicolumn{4}{|c|}{ Estadiamento tomográfico } \\
\hline$|-| \mid$ & 0 & $8 / 47$ & 0,058 \\
\hline III-IV & $6 / 100$ & $9 / 53$ & \\
\hline \multicolumn{4}{|l|}{ DHL sérico } \\
\hline normal & $2 / 34$ & $8 / 47$ & 0,66 \\
\hline elevado & $4 / 66$ & $9 / 53$ & \\
\hline
\end{tabular}

do estadiamento tomográfico $(p=0,058)$ e em relação às categorias dos níveis de $\operatorname{DHL}(p=0,66)$.

\section{Discussão}

Existe grande variação em relação à taxa de infiltração medular por $\angle D G C B$, devido ao fato de não haver uma forma clara e uniforme de estudar a $\mathrm{MO}^{(1,2,4-6)}$. Devido ao frequente envolvimento focal, Juneja et al. enfatizam a preferência para a BMO bilateral, pois demonstraram, em 20 casos de LDGCB com infiltração medular, taxa de infiltração unilateral de $50 \%{ }^{(6)}$. Outros estudos, que tam- bém comprovam a grande incidência de infiltração focal medular, demonstram que a positividade do diagnóstico está relacionada ao comprimento do fragmento estudado. Campbell et al. demonstraram, em 27 casos com infiltração medular por LDGCB, aumento da taxa de positividade de infiltração de acordo com o comprimento do fragmento. Amostras com comprimentos menores que $20 \mathrm{~mm}$ apresentaram taxa de positividade de $20 \%$ comparadas a $35 \%$ de positividade com fragmentos maiores ou iguais a 20 $\mathrm{mm}^{(3)}$. Embora haja controvérsias quanto à necessidade da BMO bilateral ou unilateral, atualmente são preconizados para o estadiamento fragmentos de no mínimo $20 \mathrm{~mm}$ de comprimento por paciente ${ }^{(4)}$. No nosso estudo, mesmo que a média do comprimento dos fragmentos por caso estudado $(27,53 \mathrm{~mm})$ tenha ultrapassado a preconizada $(20 \mathrm{~mm})$, foi encontrada taxa de infiltração medular unilateral de 21,4\%. Além disso, não houve diferenças em relação à média do comprimento dos fragmentos negativos comparados aos positivos.

Em relação aos fatores prognósticos do índice prognóstico internacional (IPI), a infiltração de $\mathrm{MO}$ não mudaria a classificação dos pacientes, pois $100 \%$ dos casos com infiltração unilateral apresentavam estadiamento radiológico III ou IV, sendo considerados de mau prognóstico.

Portanto, mesmo respeitando a sugestão do comprimento do fragmento de $\mathrm{MO}$ por caso superior a $20 \mathrm{~mm}$, a BMO bilateral foi importante, pois a taxa de infiltração unilateral atingiu $21,4 \%$, sem que essa diferença tenha sido demonstrada pelo comprimento dos fragmentos positivos ou negativos à infiltração.

\section{Referências}

1. Bain, B. Bone marrow trephine biopsy. J Clin Path, v. 54, n. 10, p. 737-42, 2001.

2. Bennett, J.M. et al. The significance of bone marrow involvement in non-Hodgkin's lymphoma: the Eastern Cooperative Oncology Group experience. J Clin Oncol, v. 4, n. 10, p. 1462-9, 1986.

3. Campbell, J.K. et al. Optimum trephine length in the assessment of bone marrow involvement in patients with diffuse large cell lymphoma, Annals of Oncology, v. 14, n. 2 , p. 273-6, 2003.

4. Cheson, B.D. et al. Report International workshop to standardise response criteria for non-Hodgkin Iymphoma, J Clin Oncol, v. 17, n. 4, p. 1244 53, 1999.

5. Conlan, M.G. et al. Bone marrow involvement by nonHodgkin's lymphoma: the clinical significance of morphologic discordance between the lymph node and bone marrow. Nebraska Lymphoma Study Group. J Clin Oncol, v. 8, n. 7, p. 1163-72, 1990.

6. Juneja, S.K., Wolf, M.M., Cooper, I.A. The value of bilateral bone marrow specimens in non-Hodgkin's Lymphoma. J Clin Path, v. 43, n. 8, p. 630-2, 1990. 